\title{
L'origine et la nature de l'innervation primitive du cour chez les embryons d'Oiseaux, (Canard et Poulet)
}

par

\section{J. SZEPSENWOL et $M^{11}$ A. BRON.}

(Travail du Laboratoire d'Anatomie de l'Université de Genève.

Directeur: M. le Professeur A. Weber.)

Avec 8 figures dans le texte.

\section{INTRODUCTION.}

On sait que chez les Oiseaux comme chez les Mammifères ce sont les nerfs vagues et sympathiques qui fournissent l'innervation du cœur; d'autre part, il a été établi que l'organe pulsatile chez ces animaux commence à battre à un stade très précoce, alors que la différenciation du canal neural n'a pas encore commencé et que les premiers filets nerveux venant des centres ne l'ont pas encore abordé; mais il n'existe pas, à notre connaissance, de travaux relatant le moment précis du début de l'innervation cardiaque. Bien que cette question ait déjà été étudiée à plusieurs reprises, elle ne semble pas encore élucidée; la plupart des auteurs ne se sont basés que sur des méthodes ordinaires de coloration; quant à ceux qui ont recouru à des techniques spécifiques pour la mise en évidence des neurofibrilles, ils n'ont eu à leur disposition qu'un nombre restreint de stades embryonnaires.

Possédant deux séries d'embryons (une centaine de Canards et une de Poulets) âgés de un à six jours, fixés de deux à quatre heures d'intervalle, imprégnés au nitrate d'argent d'après la méthode de Bielschowsky et débités en coupes sériées de 10 micra d'épaisseur, nous nous sommes proposés d'étudier l'innervation primitive du cœur. Nous allons, avant d'entrer dans notre sujet, donner un bref aperçu des travaux qui le concernent.

Rev. Suisse de Zool., T. 43, 1936. 


\section{HistoriQue.}

C'est His, Jr. (1890-1892) qui, le premier, a entrepris chez plusieurs classes d'Animaux (Poissons, Amphibiens, Oiseaux) et chez l'homme, l'étude du développement de l'appareil nerveux du cœur. Employant dans ses recherches les méthodes de coloration simple (hématoxyline-éosine), il constata que chez un embryon de Poulet de six jours l'appareil nerveux du cœur est représenté par trois nerfs qui se terminent à la base des gros vaisseaux où se trouvent déjà groupées des cellules ganglionnaires avec lesquelles ils constituent le plexus bulbaire. Au même stade, l'auteur trouva un plexus auriculaire formé de fibres et de cellules venues du mésocarde veineux. Toutefois, His pensa que la pénétration des fibres nerveuses dans le système ventriculaire le long du bulbe aortique s'effectue plus précocement que du côté auriculaire. Il arriva à la mème conclusion chez l'embryon humain, dont le cœur est également abordé par les nerfs au niveau de ses deux extrémités artérielle et veineuse. Par contre, chez les Poissons et les Amphibiens, il s'aperçut que c'est uniquement par la portion auriculaire que se fait l'innervation cardiaque.

Aвel (1912) constata, chez des embryons de Poulet imprégnés au nitrate d'argent d'après la méthode de Cajal, que c'est entre 108 et 112 heures que l'extrémité craniale du tronc artériel est abordée par les fibres et les cellules nerveuses. Chez des embryons de 120 à 140 heures, les éléments nerveux au niveau de l'appareil ventriculaire du cœur sont déjà très abondants, tandis qu'au niveau de l'extrémité auriculaire ils ne font leur première apparition qu'à 144 heures.

Perman (1924) employa dans ses recherches l'imprégnation argentique d'après la méthode de Bielschowsky; il étudia des embryons humains et de Poulet. Chez ces derniers, il trouva que l'innervation du cœur est un peu plus précoce que ne l'avaient déclaré les auteurs précédents, car il remarqua la présence des premiers éléments nerveux (fibres et cellules) au niveau de l'extrémité antérieure du tronc artériel, ainsi que dans le mésocarde veineux, déjà chez des embryons de 98 heures. Chez un embryon de quatre à cinq jours, l'auteur trouva un nombre très considérable de fibres nerveuses à proximité du cœur, et à partir de cinq à six 
jours il put les suivre tout le long du bulbe aortique et jusque dans le myocarde. En outre, à ce stade, sur le trajet des nerfs cardiaques, à la base des gros vaisseaux ainsi qu'au niveau du mésocarde veineux, se trouvent des cellules nerveuses groupées en forme de ganglions.

Dans le cœur d'un embryon humain de $13^{\mathrm{mm}}$, Perman trouva des filets nerveux aussi bien dans l'extrémité artérielle que veineuse.

Tello (1924) n'a pas fait une étude complète de l'innervation primitive du cœur chez les Oiseaux, bien qu'il se soit préoccupé de savoir si ceux-ci possèdent le nerf dépressseur de Cyon; mais il donne toutefois une description assez détaillée de la formation du nerf vague chez le Poulet. Chez celui-ci, les nerfs IX et X possèdent dans le crâne un ganglion commun correspondant aux ganglions d'Andersch et jugulaire; à partir de là, les deux nerfs se forment séparément et présentent chacun sur leur trajet un ganglion distinct. Le ganglion d'Ehrenritter du glosso-pharyngien prend naissance au niveau du troisième arc branchial, tandis que le ganglion plexiforme du vague se forme à la hauteur du quatrième arc.

$\mathrm{Au}$ cours de la croissance embryonnaire, entre cinq et six jours, les arcs aortiques III, IV et VI descendent dans le thorax avec le cœur; ils entraînent avec eux le ganglion plexiforme du pneumogastrique, qui forme autour de ces vaisseaux de nombreuses ramifications. Chez un embryon de six jours, ce ganglion est déjà situé dans le thorax, il est étendu et semi-lunaire, il embrasse dans sa concavité, à droite, les vaisseaux des IIIme, IVme et VIme arcs branchiaux (carotide, aorte et artère pulmonaire) et à gauche ceux des IIIme et VIme arcs (carotide et artère pulmonaire).

Le nerf récurrent, à ce stade, se détache de la partie inférieure du ganglion plexiforme du vague, contourne l'artère pulmonaire et remonte vers l'œesophage. Il forme autour des IIIme et IVme arcs aortiques un anneau complet, que Tello envisage comme le pendant du nerf dépresseur des Mammifères.

En somme, des travaux que nous venons de mentionner, il résulte que la pénétration des fibres nerveuses dans le cœur se fait relativement tard; le moment précis de cette pénétration, qui varie d'un auteur à l'autre, ne nous semble ni bien défini, ni exact, ainsi que nous le démontrerons plus loin.

Un autre point très important de l'innervation primitive du cœur est de savoir à quel stade du développement les cellules intracar- 
diaques font leur apparition et quelle est leur origine. Or cette question est actuellement encore très confuse et discutée.

Sans nous attacher aux anciens travaux, basés sur des colorations simples, comme ceux de His, qui n'a vu des cellules ganglionnaires au niveau du bulbe aortique que chez un embryon de Poulet de six jours et qui fait remonter leur origine aux ganglions spinaux, nous passerons à l'analyse des travaux plus récents.

AвEL (1912), ainsi que nous l'avons déjà dit, voit les premières cellules nerveuses au niveau de l'extrémité craniale du tronc artériel chez un embryon de Poulet de 108 à 112 heures, tandis qu'à l'extrémité veineuse du cœur, c'est à 104 heures qu'il les trouve pour la première fois. Pour Abel, ainsi que pour Müller (1920) qui étudia des embryons de Sélaciens, les cellules nerveuses destinées au cour émigreraient du tube neural le long du pneumogastrique. Cette opinion, qui avait déjà été soutenue auparavant par FrorieP (1907), a été reprise dernièrement par PERMAN (1924) et Kuntz (1929).

Perman (1924), ainsi que nous l'avons dit plus haut, observa les premières cellules nerveuses aussi bien à l'extrémité veineuse du cœur que dans le tronc artériel chez un embryon de Poulet de 98 heures. Ces cellules, pénétrant dans l'appareil cardiovasculaire en même temps que les fibres nerveuses. se trouveraient au niveau des terminaisons vagales.

Plusieurs auteurs contestent l'origine des plexus intracardiaques aux dépens du nerf vague.

Конn, en 1907, puis Szantroch en 1929, ont envisagé la possibilité d'une différenciation nerveuse sur place, à partir des cellules syncytiales de la région.

Tello (1924) ne rechercha pas l'origine des cellules nerveuses intracardiaques, mais en étudiant l'innervation du tube digestif chez l'embryon de Souris, il émit une hypothèse au sujet de l'origine des cellules sympathiques périphériques: les éléments du plexus d'Auerbach proviendraient, soit directement de cellules mésodermiques, soit de la différenciation sur place d'éléments médullaires émigrés de très bonne heure, avant toute manifestation neurofibrillaire. Alors que le pneumogastrique se termine, chez un embryon de Souris de $4 \mathrm{~mm}$, dans la moitié supérieure de l'estomac, la portion duodénale du tube digestif est déjà pourvue de cellules nerveuses bien différenciées; or, cette portion de l'intestin antérieur 
est, à ce moment, encore complètement dépourvue de fibres centrifuges, les nerfs splanchniques n'étant pas formés.

Tello retrouva cette indépendance des plexus nerveux du duodénum par rapport aux nerfs intrinsèques d'une manière évidente chez le Poulet qui, dès trois jours, révèle dans la partie postérieure de l'intestin antérieur des cellules fusiformes à réseau neurofibrillaire, lesquelles donnent naissance, vers trois jours et demi, à un plexus bien organisé; celui-ci ne se met en contact avec les terminaisons du pneumogastrique qu'au cours du quatrième et du cinquième jour.

TELLO, ne trouvant nulle part les signes d'une migration cellulaire, ni le long du nerf vague, ni ailleurs, conclut donc que, si migration il y a, celle-ci se fait du moins de très bonne heure, lorsque les cellules sont encore exemptes de toute manifestation nerveuse.

Van Campenhout (1931) fit faire un pas en avant à la théorie de Tello. Il rechercha expérimentalement le développement du système nerveux périphérique chez le Poulet et utilisa les méthodes ordinaires de coloration aussi bien que l'imprégnation argentique d'après la méthode de Bielschowsky.

Van Campenhout, d'accord avec Tello, a constaté que la chaîne prévertébrale du sympathique tire son origine de la crête ganglionnaire et que sa formation est un processus continu, débutant à la fin du deuxième jour et atteignant son développement définitif à la fin du sixième jour. Pour ce qui en est des premières formations nerveuses de la région cardiaque, il remarqua que les nerfs pneumogastriques forment des plexus, riches en cellules, périœsophagien, péribronchique et donnent à ce moment naissance à quelques rameaux cardiaques, avant que les fibres et les éléments sympathiques puissent être suivis. Les cellules de ces plexus du vague n'auraient rien à voir avec l'origine de ce nerf; elles proviendraient, soit de cellules mésenchymateuses différenciées sur place, soit d'éléments sympathiques émigrés des ganglions rétro-aortiques le long des branches viscérales.

Van Campenhout base son hypothèse sur les expériences suivantes: il extirpe, chez des embryons de Poulet de 32 heures, le bulbe rachidien, de manière à empêcher la formation du nerf vague; malgré l'absence de ce nerf, les plexus nerveux périphériques (œsophagien, gastrique, hépatique, cardiaque, etc.) se forment comme normalement. En outre, par le moyen des greffes chorio- 
allantoïdiennes, l'auteur trouva que des fragments abdominaux d'embryons de plus de 56 heures donnent naissance à des plexus nerveux intraviscéraux; par contre, ces derniers ne se forment pas à partir de fragments embryonnaires de moins de 56 heures. C'est cette deuxième expérience qui amena Van Campenhout à exclure la différenciation sur place de cellules mésenchymateuses. Cet auteur croit plutôt à une migration de neuroblastes sympathiques qui s'accomplirait au début du troisième jour.

En somme, des travaux que nous venons de signaler, ressortent trois théories absolument opposées, l'une admettant l'origine vagale et médullaire des cellules nerveuses du cœur, l'autre l'origine mésenchymateuse, la troisième enfin une origine sympathique ganglionnaire. Certains auteurs ont essayé de concilier ces divers avis.

Kuntz (1929) étudia de nombreux Mammifères et une série d'embryons humains. Chez ceux-ci, il arriva à la conclusion que les plexus pulmonaires, cardiaques et entériques sont formés, au début de leur apparition, par des fibres et des cellules vagales; mais il n'est pas exclu que, peu après, ils ne soient pénétrés par des fibres et des cellules sympathiques, d'ailleurs peu nombreuses.

Uchid a (1927) prétend par contre que le déplacement des cellules sympathiques pour former les plexus entériques se fait avant que les cellules vagales n'atteignent l'intestin; celles-ci ne se mêleraient que secondairement à ce plexus; l'inverse se produirait pour les plexus œsophagien et gastrique, formés primitivement d'éléments médullaires émigrés le long du pneumogastrique, auxquels se mêleraient plus tard quelques fibres et cellules sympathiques.

\section{Recherches PERSONNELLES.}

Avant de commencer l'étude des fibres propres sympathiques et para-sympathiques du cœur, nous croyons utile de parler tout d'abord de la formation du nerf vague et de son développement.

Nous ferons notre description d'après l'embryon de Canard qui, présentant sur le Poulet l'avantage de se développer plus lentement, montre par conséquent plus nettement les étapes de la différenciation nerveuse. 


\section{Embryons de Canard.}

Fibres nerveuses:

Chez un embryon de Canard de 65 à 66 heures d'incubation, le tube neural montre le début de sa différenciation. Seules quelques cellules périphériques situées à l'extrémité antérieure du canal médullaire possèdent déjà des prolongements qui, du reste, ne franchissent pas la limitante externe, de sorte qu'il ne peut encore être question de la racine motrice de la dixième paire de nerfs craniens; par contre, l'amas de cellules situé tout de suite en arrière de la vésicule auditive et qui, organisé en un ganglion commun pour les nerfs IX et $\mathrm{X}$, représente la somme des ganglions d'Andersch et jugulaire, révèle déjà la présence d'éléments bipolaires munis de prolongements assez longs, dont les proximaux, chez un embryon de 70 heures, franchissent déjà la limitante externe et pénètrent dans la vésicule cérébrale postérieure. Quant à la racine motrice du vague, c'est à ce moment qu'elle commence à s'ébaucher; mais, de la partie ventrale du rhombencéphale, n'émergent que quelques fibres isolées qui se perdent dans le mésenchyme, tandis que par contre les filaments qui sortent de la partie postérieure de la moelle allongée en suivant le trajet de la racine sensitive sont plus nombreuses; il s'agit là des fibres "radiculaires motrices postérieures » (TELLO).

A 75 heures, le complexe du glosso-pharyngien-vague présente d'importants changements et son trajet se complique. On peut, à ce stade, le diviser en deux portions, l'une horizontale, l'autre verticale; la première, qui comprend le tronc commun des nerfs IX et X, et qui émerge du rhombencéphale en arrière de la vésicule auditive, chemine parallèlement à l'ectoderme à travers le mésenchyme; sur son parcours on distingue l'amas de neuroblastes qui, comme nous l'avons déjà dit, représente l'ensemble des ganglions d'Andersch et jugulaire. Cette portion horizontale du glosso-pharyngien-vague se prolonge jusqu'à la face dorsale de la veine céphalique (jugulaire interne); à ce niveau, elle se coude pour prendre une direction verticale; c'est là que prend naissance la deuxième portion; celle-ci se divise tout de suite en deux branches, dont l'une représente le nerf vague proprement dit et l'autre le nerf glosso-pharyngien. Ce dernier s'engage dans l'interstice qui sépare la veine et l'artère céphaliques pour se diriger vers le IIIme arc branchial, tandis que 
le nerf vague continue son chemin en dehors et en arrière de la veine céphalique, et au voisinage immédiat de l'ectoderme; très court, le pneumogastrique se termine par quelques fibres au niveau d'un groupe de cellules qui marquent un début de différenciation neurofibrillaire et sont destinées à former plus tard le ganglion plexiforme du nerf vague, thoracique chez les Oiseaux. Cette ébauche ganglionnaire est disposée tellement près de l'ectoderme qu'il est impossible de distinguer une limite nette de séparation; elle se trouve située dans le IVme arc branchial, encore mal dessiné, et se termine au niveau de l'extrémité supérieure de l'aorte descendante, c'est-à-dire à la moitié du pharynx.

Chez un embryon de 80 heures, le ganglion thoracique du vague, qui a beaucoup augmenté de volume, se présente sous la forme d'un croissant qui embrasse dans sa concavité la veine céphalique; toutefois, cette masse ganglionnaire est encore accolée à l'ectoderme et, par endroits, il est difficile de distinguer la zone de démarcation autrement qu'en se basant sur l'aspect des cellules qui, dans le ganglion, ont déjà revêtu leur caractère neuronal, grâce à leur forme bipolaire et l'apparition d'un réseau neurofibrillaire. Il est à remarquer qu'à cet âge la portion préganglionnaire du nerf vague est encore très mince, pauvre en fibres (deux à trois), tandis que la portion postganglionnaire, bien qu'à peine ébauchée, révèle déjà toutefois un nombre plus considérable de courtes fibres, engagées en arrière du pharynx.

Au cours des heures suivantes, jusqu'à 84 heures, la portion préganglionnaire (thoracique) du pneumogastrique ne manifeste pas de grands changements. En effet, le pinceau nerveux médullaire ne compte pas plus de cinq à six fibres, qui se perdent à l'intérieur du ganglion plexiforme qui, par contre, progresse activement. Dès 84 heures, celui-ci est nettement séparé de l'ectoderme, bien individualisé, et se présente, sur des coupes transversales, sous la forme d'une masse ronde disposée en avant et en dehors de la veine céphalique. Ses éléments, tous différenciés, envoient des prolongements jusqu'à l'extrémité postérieure du pharynx. Peu avant son ganglion thoracique, le nerf vague envoie quelques fibres dans la direction du ganglion d'Ehrenritter, qu'elles atteindront aux environs de 88 heures. Du ganglion d'Ehrenritter lui-même se détache un groupe de fibres destinées à constituer le nerf laryngé supérieur, quelques-uns de ces filaments se dirigent vers l'artère 
céphalique, qu'elles abordent par sa paroi ventrale et dans l'endothélium de laquelle elles s'épuisent par un bouton terminal dès 84 heures. Nous sommes donc là en présence du premier contact du système nerveux avec l'appareil vasculaire. Quant au nerf laryngé supérieur, il atteint la paroi pharyngienne d'un embryon à 88 heures. D'autre part, de l'extrémité supérieure du ganglion thoracique du vague, se détachent quelques filaments qui se perdent dans la paroi de la veine céphalique, directement au contact de l'endothélium, dès 84 heures, réalisant ainsi le premier contact du nerf vague avec le système vasculaire.

La Figure $I$ représente une coupe transversale d'un embryon de Canard de 84 heures passant au niveau du pharynx. On y voit l'extrémité supérieure du ganglion thoracique du vague, qui envoie un ou deux filaments dans la paroi de la veine céphalique. Le ganglion d'Ehrenritter du glossopharyngien, situé plus ventralement, donne naissance à un pinceau de fibres dont quelques-unes abordent la paroi de l'artère céphalique et s'y épuisent par des boutons terminaux.

A 88 heures, la portion préganglionnaire du nerf vague, qui a pris de l'importance, n'est cependant pas de la même épaisseur que la portion postganglionnaire; elle se

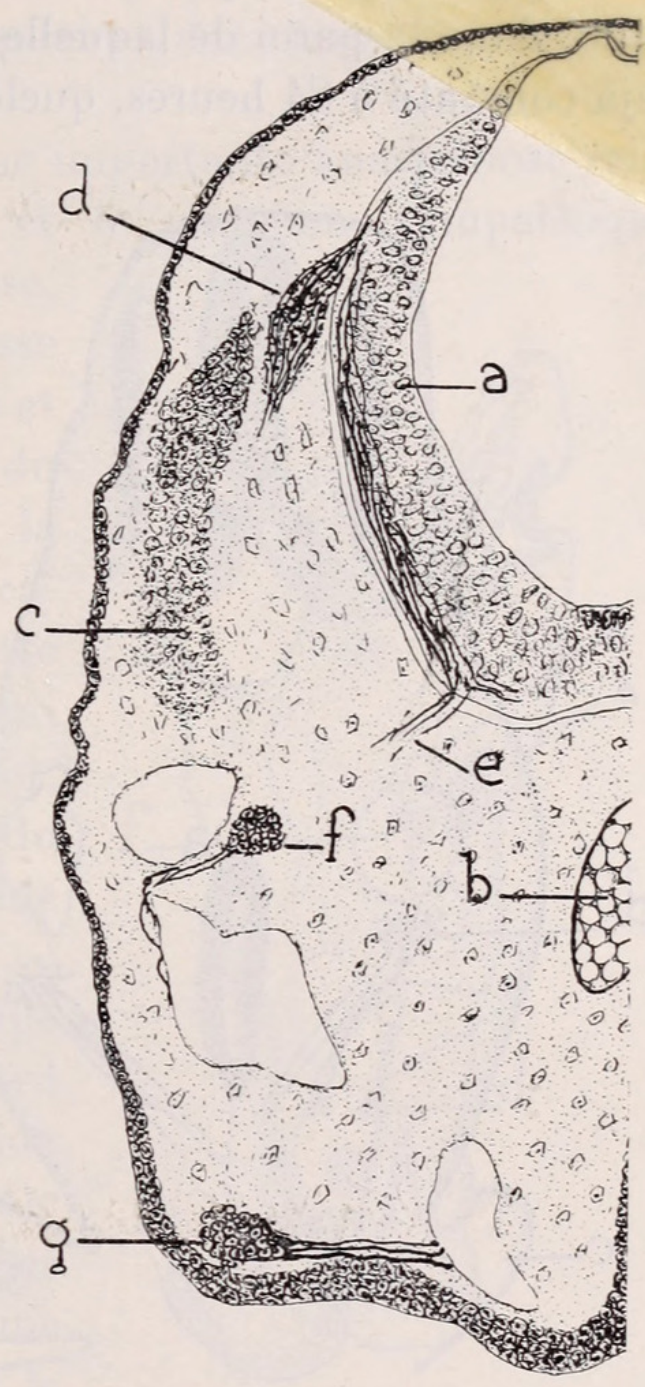

Fig. I. - Coupe transversale au niveau du rhombencéphale d'un embryon de Canard de 84 heures.

Dessin à la chambre claire d'une coupe grossie 100 fois de la région rhombencéphalique: $a)$ rhombencéphale; $b$ ) corde dorsale; c) somites; d) ganglion commun des $\mathrm{Ix}_{\mathrm{me}}$ et $\mathrm{x}_{\mathrm{me}}$ paires; e) racine motrice $\mathrm{du}$ vague; f) ganglion plexiforme, d'où partent des fibres qui se terminent dans la paroi de la veine céphalique; g) ganglion d'Ehrenritter, qui donne naissance au nerf laryngé supérieur et dont quelques fibres se terminent dans la paroi de l'artère céphalique. présente toutefois déjà comme un faisceau bien fourni, surtout au 
voisinage du ganglion thoracique; celui-ci, qui s'est écarté considérablement de l'ectoderme et s'en trouve séparé par une épaisse couche de mésederme, s'est placé tout de suite en avant de la veine céphalique, dans la paroi de laquelle se terminent, ainsi que nous l'avons déjà constaté à 84 heures, quelques-uns de ses prolongements.

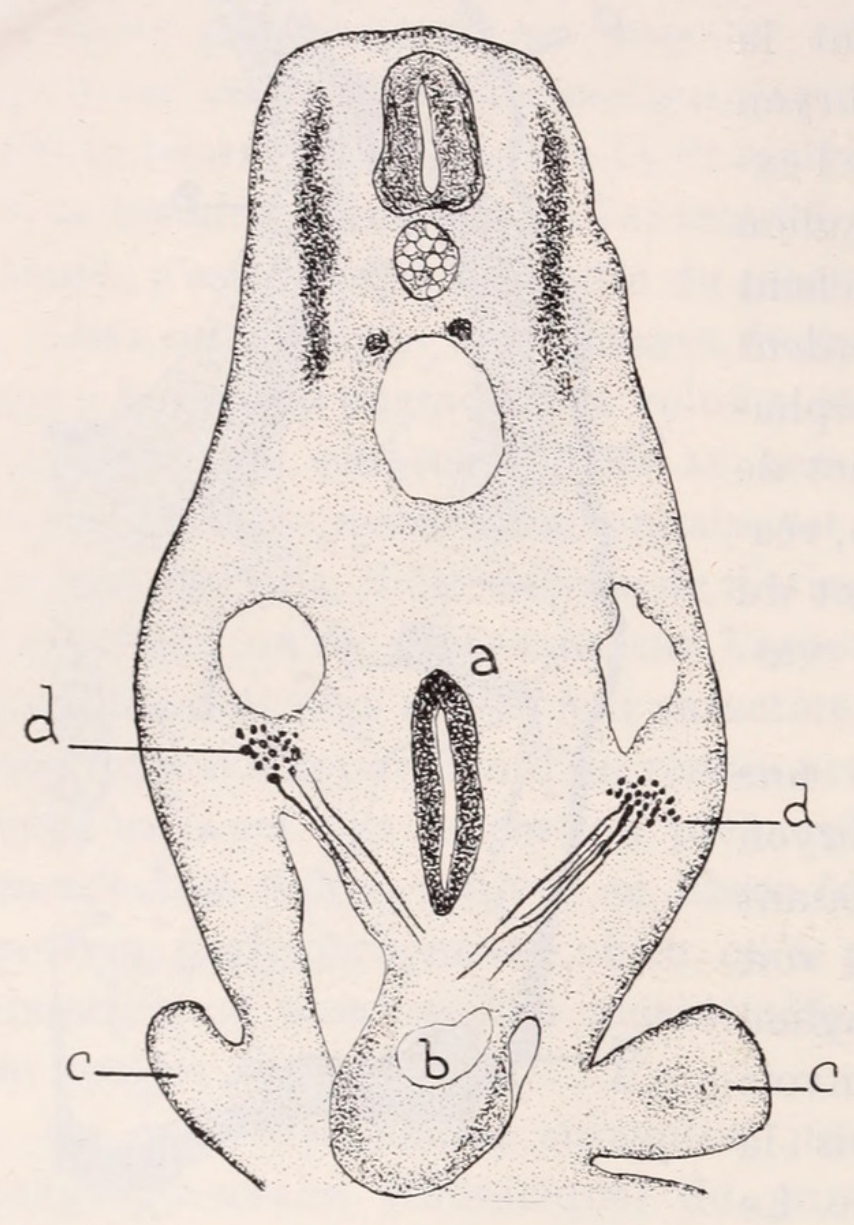

Fig. II. - Coupe transversale de la région cardiaque d'un embryon de Canard de 90 heures.

Dessin à la chambre claire d'une synthèse de plusieurs coupes successives grossies 55 fois, dans la région du bulbe aortique: $a$ ) tube digestif; b) bulbe aortique; $c$ ) arcs branchiaux; d) nerfs vagues gauche et droit, qui donnent naissance à quelques fibres pour le bulbe aortique.

A 90 heures, le nerf vague, déjà très long, descend plus bas que le tronc artériel et se termine à la hauteur de la trachée; au cours de son trajet, il abandonne quelques fibres qui se perdent au voisinage du bulbe aortique, ainsi qu'on le voit sur la Figure II; celle-ci représente une coupe transversale d'un embryon de Canard de 90 heures, à la hauteur du bulbe aortique, dans la région duquel se perdent quelques fibres vagales.

A 94 heures, le ganglion plexiforme du vague, légèrement entraîné en arrière de la jugulaire interne, se bifurque à son extrémitécaudale en deux branches, qui représentent l'ébauche des deux faisceaux du pneumogastrique décrits par Couvreur (1892) chez l'adulte. L'un de ces deux rameaux, ventral, très court, longe la paroi postérieure du pharynx; il pénétrera ultérieurement dans le bulbe aortique; l'autre, plus dorsal, situé entre la veine jugulaire et l'ectoderme, descend le long de l'œsophage et se termine légèrement 
en avant du bulbe aortique. A part ces deux branches assez importantes, se détachent du ganglion plexiforme du vague quelquess fibres qui se dirigent transversalement en passant en avant des vaisseaux céphaliques (carotide et jugulaire), dans la paroi desquelles quelques-unes se terminent.

Chez un embryon de 98 heures, une importante anastomose relie le ganglion thoracique du vague et le ganglion sympathique cervical supérieur. Cette anastomose, composée de fibres et de cellules, glisse le long de la paroi latérale de l'aorte et va rejoindre le ganglion plexiforme du pneumogastrique en passant entre la veine céphalique à l'extérieur et la carotide à l'intérieur; autour de cette artère, elle forme un plexus assez compliqué dont les cellules, par leur aspect, rappellent les éléments de la chaîne du sympathique; nous aborderons l'étude de ces cellules plus loin.

La Figure III représente une coupe transversale d'un embryon de Canard de 98 heures, passant dans la région du ganglion thoracique du vague. De ce dernier, on voit se détacher quelques fibres, qui se dirigent vers le quatrième arc aortique et se perdent dans le plexus nerveux périvasculaire, dans lequel on reconnait de nombreux éléments originaires de la partie antérieure de la chaîne du sympathique, ainsi qu'en témoignent les neuroblastes situés tout le long de la paroi latérale de l'aorte, et qui semblent en voie de migration. Ce plexus nerveux est représenté à un plus fort grossissement sur la Figure $I V$.

En outre, à 98 heures, le ganglion plexiforme du vague, situé en dehors

des ares aortiques, donne naissance à une branche nerveuse, en-

Fig. III. - Coupe transversale de l'extrémité antérieure d'un embryon de Canard de 98 heures.

Dessin à la chambre claire d'une synthèse de trois coupes successives grossies 40 fois : a) ganglion thoracique du vague; $b$ ) carotide; c) aorte; $d$ ) ganglion sympathique cervical supérieur; e) cellules sympathiques émigrant le long de l'aorte; f) plexus péricarotidien formé par l'anastomose vagosympathique. 
core peu fournie, qui se dirige transversalement vers l'extrémité supérieure de l'œsophage en passant au-dessous du quatrième

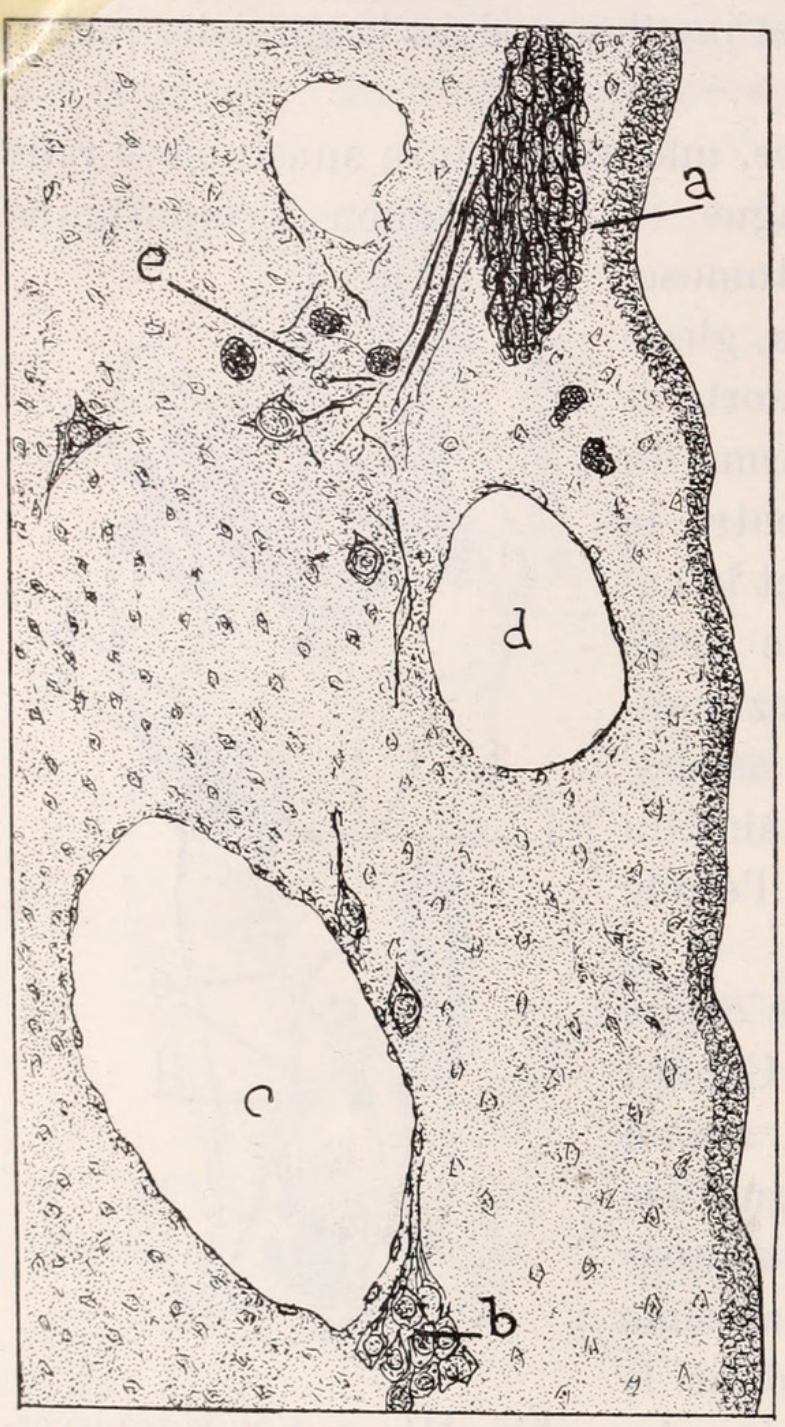

Fig. IV. - Région de l'anastomose vagosympathique de la figure III à un plus fort grossissement.

Dessin à la chambre claire d'une synthèse de trois coupes successives grossies 185 fois: a) ganglion thoracique du vague; b) ganglion sympathique cervical supérieur; $c$ ) aorte; $d$ ) veine céphalique; e) plexus nerveux péricarotidien formé par des fibres vagales et des cellules sympathiques. avant d'atteindre la paroi gastrique, s'anastomose avec le pneumogastrique du côté opposé en formant le plexus périœsophagien. En ce qui concerne les branches cardiaques du nerf vague, excepté du nerf récurrent. Le nerf vague lui-même se termine, à ce moment, en se divisant en deux branches principales dont la plus importante, interne, se rapproche de la ligne médiane pour longer l'œsophage et se répartir à son pourtour ainsi qu'au voisinage des bronches; l'autre branche, moins considérable, mais cependant assez fournie, se dirige ventralement et se termine à la base du bulbe aortique. Il est à noter qu'à ce stade on ne trouve point de cellules nerveuses au voisinage de la portion artérielle du cœur.

Chez des embryons plus avancés, jusqu'à 104 heures, il ne se produit pas de grands changements dans l'innervation $d u$ cœur et la distribution du nerf vague, si ce n'est que ce dernier s'allonge de plus en plus; à 106 heures, ses branches broncho-pulmonaires atteignent déjà l'ébauche du poumon, et la branche du tube digestif la partie supérieure de l'estomac; cette dernière branche,

arc aortique; il s'agit ici 
celles qui ont pénétré dans le système artériel aux environs de 90 heures, c'est à l'âge de 106 heures que quelques rameaux destinés à s'introduire dans l'appareil auriculaire à 118 heures se détachent des nerfs du tube digestif, légèrement au-dessus de la fusion des deux vagues.

La Figure V représente une coupe transversale de la région cardiaque d'un embryon de Canard de 118 heures. On y voit les deux nerfs vagues, situés de chaque côté de l'œsophage, dont quelques fibres se détachent, contournent l'ébauche pulmonaire, les bronches et s'engagent dans l'espace interauriculaire du cœur.

En somme, les premières fibres du nerf vague abordent le système vasculocardiaque chez un embryon de Canard de 88 heures; c'est le tronc artériel (arcs aortiques) qui est pénétré tout d'abord ; ensuite c'est le bulbe aortique (embryons de 90 à 98 heures) et tout à la fin e'est la portion auriculaire (embryons de 112 à 118 heures).

En ce qui concerne le système nerveux sympa-

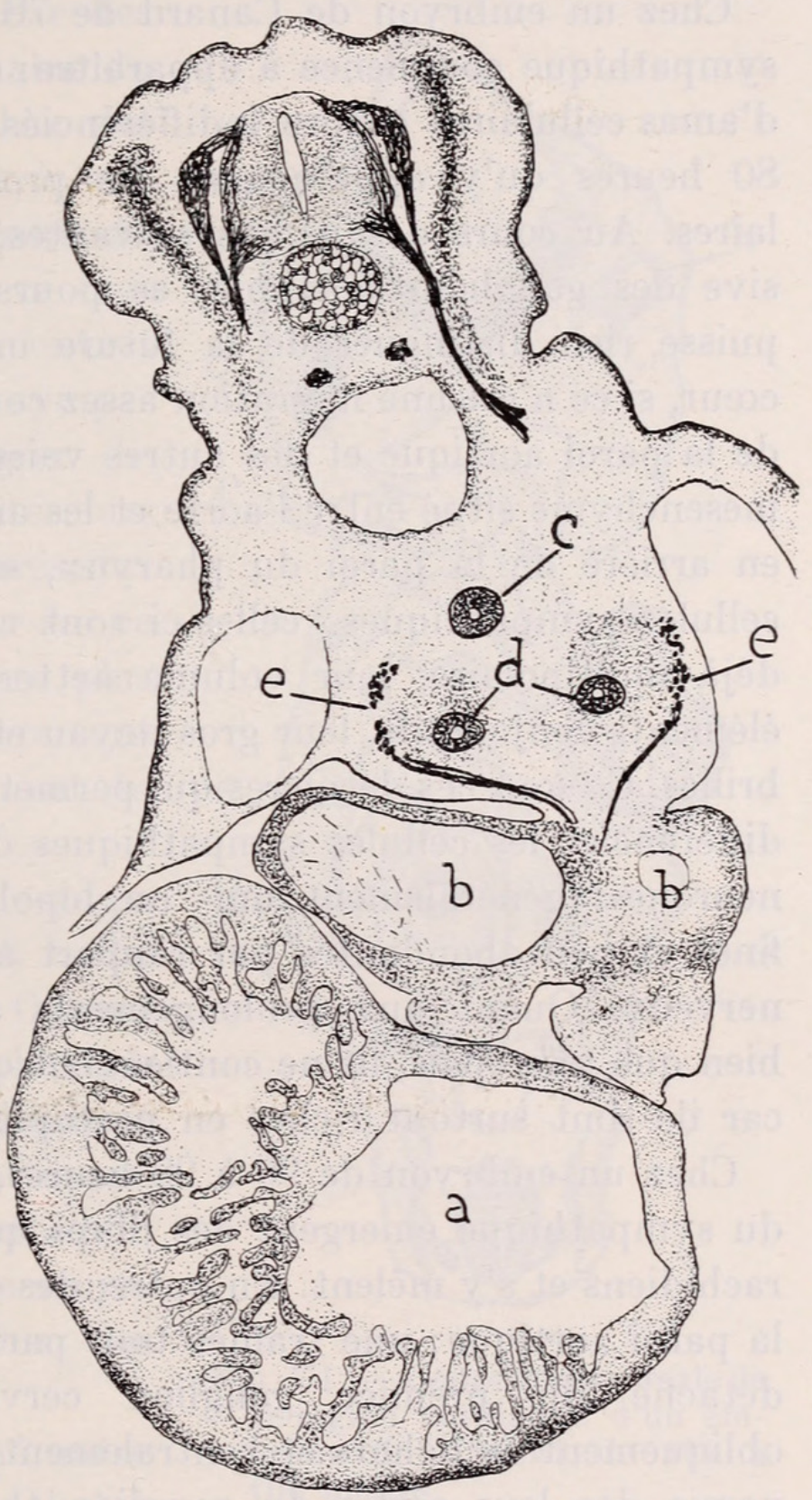

Fig. V. - Coupe transversale de la région cardiaque d'un embryon de Canard de 118 heures.

Dessin à la chambre claire d'une synthèse de trois coupes successives grossies 55 fois: a) ventricules; $b$ ) oreillettes; c) tube digestif; d) bronches; e) nerfs vagues gauche et droit, dont deux branches se terminent dans la portion auriculaire du cœur. thique du cœur, sa pénétration parait se faire un peu plus tardivement. 


\section{Cellules nerveuses:}

Chez un embryon de Canard de 70 à 75 heures, la chaîne du sympathique commence à apparaître; elle est formée à ce moment d'amas cellulaires encore indifférenciés; ce n'est qu'à l'âge de 78 à 80 heures qu'y apparaissent les premiers éléments neurofibrillaires. Au cours des heures suivantes, la différenciation progressive des ganglions aortiques se poursuit activement sans qu'on puisse rien distinguer de la future innervation sympathique du cœur, si ce n'est une migration assez considérable de cellules le long de la paroi aortique et des autres vaisseaux, de sorte que, dans le mésenchyme situé entre l'aorte et les arcs branchiaux tout de suite en arrière de la paroi du pharynx, se trouvent disséminées des cellules sympathiques; celles-ci sont caractérisées par leur forme déjà multipolaire, leur volume nettement supérieur à celui des éléments conjonctifs, leur gros noyau et par la présence de neurofibrilles. Ce sont ces dernières qui permettent, dès leur apparition, de différencier les cellules sympathiques des centrales et surtout des neurones (généralement uni- ou bipolaires) du vague; elles sont fines et peu abondantes par rapport à celles des autres éléments nerveux. Quant aux prolongements des cellules sympathiques, bien que très épais, ils ne contiennent qu'une à deux neurofibrilles, car ils sont surtout riches en protoplasme.

Chez un embryon de 96 à 98 heures, des ganglions de la chaîne du sympathique émergent des fibres qui se dirigent vers les nerfs rachidiens et s'y mêlent. En outre, des cellules et des fibres longent la paroi aortique; une traînée tout particulièrement importante se détache du premier ganglion cervical supérieur, se dirige obliquement en dehors et ventralement et rejoint les fibres du nerf vague dès leur sortie du ganglion thoracique plexiforme. Cette anastomose vago-sympathique se fait derrière la paroi dorsale du pharynx, au voisinage du quatrième arc aortique qu'elle encercle dans un riche plexus. Nous tenons à remarquer que c'est dans les mailles de ce plexus et au contact de la carotide que viennent se fixer des cellules d'aspect tout-à-fait spécial (grandes, à noyau volumineux, violemment colorées en brun-noir par le nitrate d'argent), qui vont constituer l'ébauche de la glandule carotidienne, ainsi qu'on le constate chez des embryons plus âgés. (L'un de nous, Szepsenwol, vient d'en faire la description). 
Les cellules sympathiques qui, au cours du quatrième jour, se sont engagées dans le mésenchyme au pourtour des arcs aortiques et qui semblent se propager à partir des ganglions supérieurs de la chaîne du sympathique, atteignent, chez des embryons de 102 à 106 heures, le voisinage de l'extrémité supérieure du bulbe aortique. C'est donc à ce moment que s'établit le premier contact du cœur avec les éléments sympathiques, qui semblent émigrer directement des ganglions antérieurs en se propageant le long des arcs aortiques. Nous tenons à insister sur ce point, car, comme on le verra plus loin, le cœur reçoit ultérieurement des neuroblastes qui proviennent du pourtour du tube digestif.

La Figure VI représente une coupe transversale à la hauteur du bulbe -aortique chez un embryon de Canard de 104 heures. Au niveau de l'extrémité antérieure de l'aorte, à l'endroit où celle-ci forme les arcs aortiques, on trouve une cellule sympathique; celle-ci fait suite à une série de neuroblastes disposés au pourtour des vaisseaux branchiaux.

Le plexus cardique est formé, outre les cellules provenant de la chaîne du sympathique cervical qui abordent le cœur par son extrémité aortique, par des éléments qui se propagent, à partir du pourtour du tube digestif, dans la direction de la portion auriculaire du cœur.

Dans la paroi du tube digestif, les premiers éléments nerveux apparaissent entre 90 et 96 heures. A l'âge de 104 à 106 heures, le 
plexus sympathique duodénal se dessine nettement jusqu'au niveau de l'artère omphalo-mésentérique; à 118 et 120 heures, ses éléments sont devenus très nombreux; quelques-uns d'entre eux se dirigent jusque vers la portion veineuse du cœur dans laquelle, dès

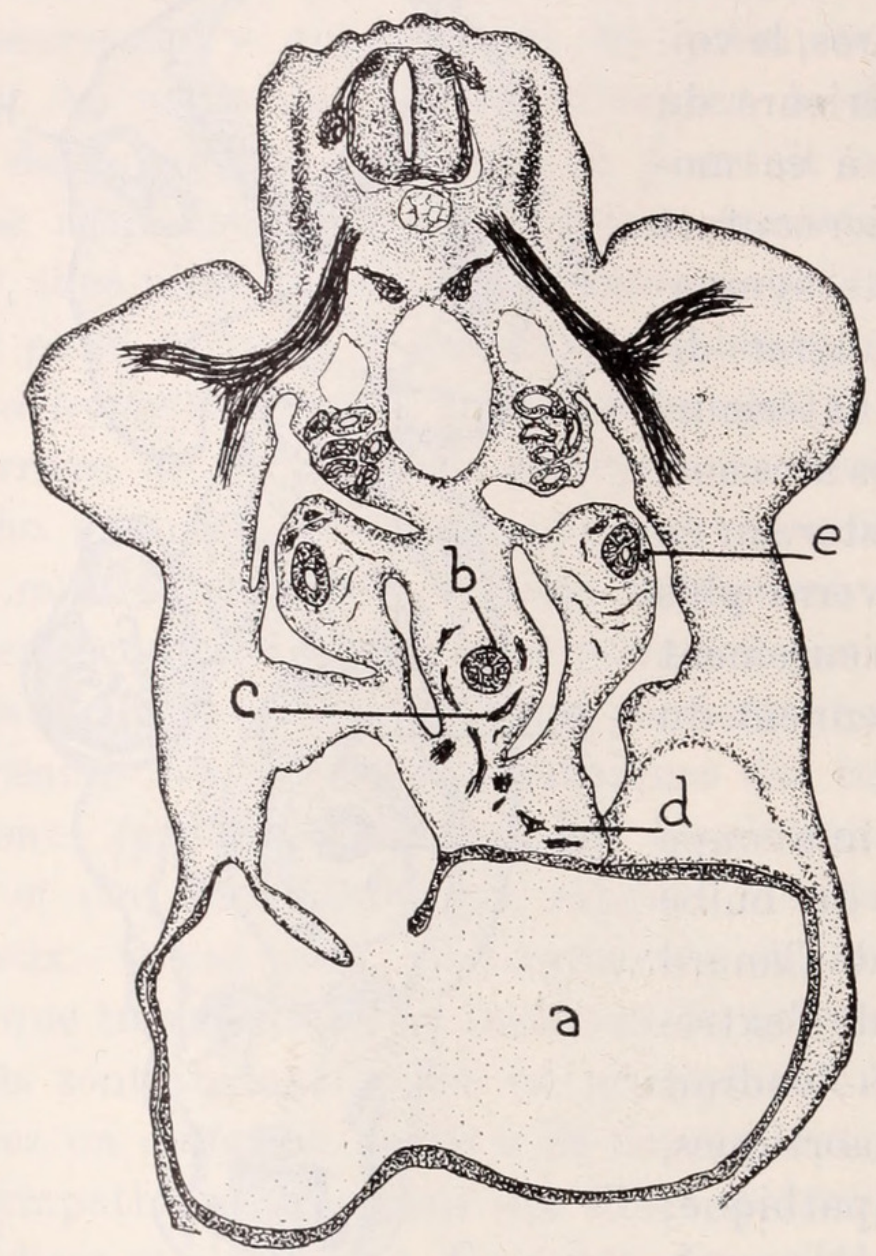

Fig. VII. - Coupe transversale de la région cardiaque d'un embryon de Canard de 132 heures.

Dessin à la chambre claire d'une coupe grossie 30 fois: $a$ ) oreillette; $b$ ) tube digestif ; $c$ ) plexus nerveux périintestinal; $d$ ) cellules ganglionnaires cardiaques; e) bronches avec plexus péribronchique.

132 heures, on trouve une ébauche ganglionnaire formée d'une dizaine de sympathicoblastes bien différenciés.

La Figure VII représente une coupe transversale de la région cardiaque d'un embryon de Canard de 132 heures. Des deux nerfs vagues situés au pourtour del'œsophage, des branches bien fournies se détachent; elles vont se terminer dans le tissu conjonctif de la région auriculaire du cœur. A ce niveau, on trouve un groupe de cellules sympathiques, dont une seule est visible sur cette coupe; cette cellule est encore plus nette sur la Figure VIII, qui montre la même région

photographiée à un fort grossissement; elle est multipolaire, volumineuse, avec un gros noyau et pauvre en neurofibrilles, dont on ne voit qu'une seule sur la microphoto. A côté de ce neurone sympathique se trouve le prolongement d'une autre cellule située sur la coupe précédente.

En somme, le système sympathique cardiaque chez le Canard apparaît un peu plus tard que celui du nerf vague. Alors que 
ce dernier aborde les vaisseaux aortiques chez un embryon de 88 heurès, les fibres provenant du ganglion sympathique cervical supérieur n'y arrivent qu'entre 96 et 98 heures. Quant aux ganglions cardiaques, leur première apparition se fait à l'âge de 106 heures à

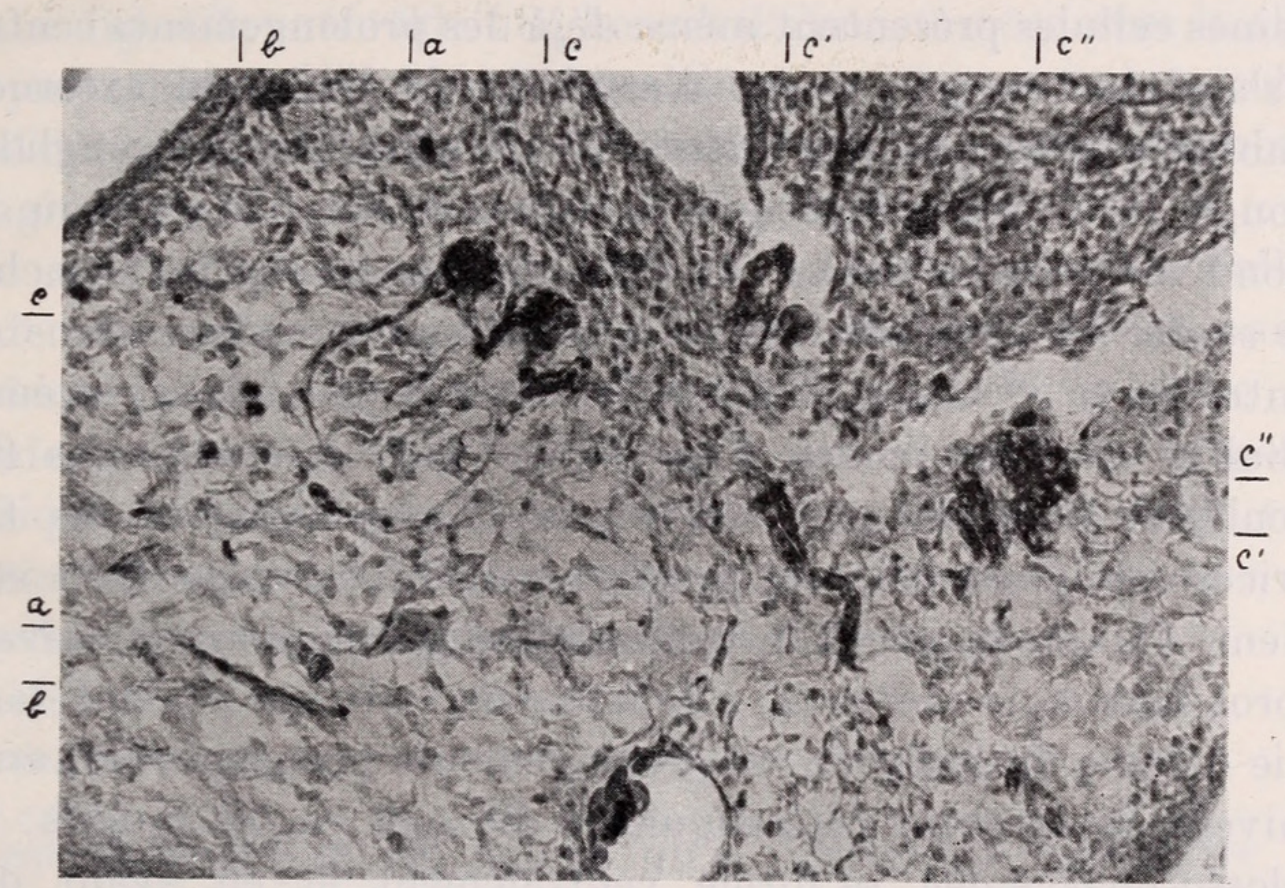

Fig. VIII. - Coupe transversale de la région périauriculaire de la figure VII à un plus fort grossissement.

Microphoto d'une coupe grossie 200 fois. a) cellule ganglionnaire cardiaque. b) prolongement d'une autre cellule située sur la coupe précédente; $c^{\prime}$ ), $c^{\prime \prime}$ ) fibres vagales pour le cour.

l'extrémité ventriculaire et de 120 heures à l'extrémité auriculaire, que les rameaux du pneumogastrique ont déjà toutes deux largement envahie.

\section{Embryons de Poulet.}

Chez le Poulet, nous avons observé, au sujet de l'innervation primitive du cœur, exactement les mêmes phénomènes que chez le Canard; nous allons en donner un bref aperçu.

Fibres nerveuses:

On sait que, chez le Poulet, la différenciation nerveuse commence aux environs de 45 à 48 heures (Tello, Gerini) et que vers 50

Rev. Suisse de Zool., T. 43, 1936. 
à 52 heures émergent du canal neural les premières fibres destinées à former les futures racines motrices. Chez un embryon de 52 heures, la racine motrice du nerf vague n'est pas encore formée, tandis que le ganglion commun pour la IXme et la $X^{m e}$ paires (Andersch et jugulaire) montre un début de différènciation neurofibrillaire; certaines cellules présentent même déjà des prolongements centraux qui s'arrêtent un peu avant d'atteindre la limitante externe du rhombencéphale; par son extrémité périphérique, le ganglion se prolonge ventralement jusqu'à la hauteur du pharynx où il se confond avec une placode ectodermique de la région branchiale. A ce stade, les arcs branchiaux commencent à se dessiner.

Entre 56 et 58 heures, la branche motrice du nerf vague émerge du bulbe en deux points, l'un dorsal, par où sortent les fibres radiculaires motrices postérieures, et l'autre ventral; ces fibres motrices rejoignent déjà le ganglion sensitif correspondant et s'y perdent. Quant au ganglion commun du glosso-pharyngien-vague, ses prolongements centraux, à ce moment, ont déjà pénétré dans la partie dorsale du bulbe et ses prolongements périphériques, arrivés au niveau de la veine céphalique, se divisent en deux nerfs: l'un, le glosso-pharyngien, se dirige ventralement et en avant de la jugulaire; l'autre, le pneumogastrique, chemine dorsalement et extérieurement par rapport à la veine. Peu après leur séparation, les deux nerfs présentent sur leur trajet des ganglions; celui d'Ehrenritter, attribué au glosso-pharyngien, est plus ventral que celui du vague, dit plexiforme ou thoracique, situé plus dorsalement. Ce dernier ganglion, encore très peu marqué à cet âge, se confond avec l'ectoderme épaissi à cet endroit et dont il n'est pas encore complètement séparé; mais, dès 60 à 64 heures, il se dessine assez nettement et les fibres qui en émergent atteignent déjà la partie inférieure du pharynx.

Chez un embryon de Poulet de 64 à 66 heures, se détachent du ganglion thoracique du vague des fibres qui vont se terminer en plexus autour de la carotide; d'autres se rendent au ganglion d'Ehrenritter du glosso-pharyngien, avec une branche duquel elles contribuent à former le nerf laryngé supérieur.

A 68 heures, le nerf vague chemine le long de la paroi postérieure du pharynx jusqu'à l'extrémité supérieure de l'œsophage. A la base du pharynx, une branche s'en détache, longe le quatrième arc aortique jusqu'à l'extrémité supérieure du bulbe aortique où il se termine 
au niveau où celui-ci donne naissance aux vaisseaux branchiaux. C'est donc entre 64 et 68 heures que le pneumogastrique prend contact avec le système vasculo-cardiaque (artères branchiales et bulbe aortique).

A partir de ce moment, le vague s'accroît rapidement en longeant le tube digestif; entre 80 et 84 heures il s'anastomose avec la chaîne du sympathique déjà bien différenciée grâce à une traînée cellulofibrillaire qui aboutit à l'extrémité inférieure du ganglion thoracique: il s'agit là de la première union vago-sympathique.

$\mathrm{Au}$ cours des heures suivantes, le pneumogastrique s'allonge rapidement; chez des embryons de 92 heures, il atteint par son extrémité périphérique l'ébauche hépatique; on constate qu'il abandonne sur son trajet, un peu avant d'atteindre la portion gastrique du tube digestif, un pinceau nerveux qui se dirige transversalement dans la direction de la portion veineuse du cœur, dans laquelle on trouve les premières fibres à 96 heures.

\section{Cellules nerveuses:}

C'est entre 64 et 66 heures qu'à l'extrémité antérieure d'un embryon de Poulet apparaissent les premiers éléments de la chaîne du sympathique en différenciation neurofibrillaire. Dès 75 heures, on constate que des cellules sympathiques émigrent en direction ventrale tout le long de la paroi aortique. C'est au même moment qu'au pourtour du tube digestif on remarque les premiers neuroblastes. Entre 80 et 84 heures, s'effectue l'importante anastomose vago-sympathique que nous avons déjà signalée. Quant à la première pénétration des cellules sympathiques dans le cœur, elle débute à 84 heures avec leur apparition au voisinage du bulbe aortique. Ce n'est qu'entre 105 et 110 heures que la portion veineuse entre en contact avec les éléments sympathiques; ceux-ci tirent leur origine du plexus intestinal, tandis que ceux de l'extrémité artérielle émigrent du ganglion cervical supérieur.

\section{Discussion.}

Dans nos recherches nous avons eu l'intention d'élucider deux points: $1^{0}$, le moment précis de la pénétration dans le cœur des fibres nerveuses; $2^{\circ}$, leur nature. 
Les auteurs qui se sont occupés de l'innervation primitive du cœur ont émis à ce sujet des avis très différents et ne l'ont aperçue qu'à des stades tardifs; cependant tous étaient d'accord, ce que nous venons également de constater, que c'est le nerf vague qui aborde en premier le cœur et secondairement le système sympathique; mais la pénétration des fibres de ce dernier suit de beaucoup plus près celle des fibres vagales que cela n'a été admis jusqu'à présent, puisque nous venons de constater qu'une anastomose vago-sympathique s'établir dès 98 heures chez le Canard et 84 heures chez le Poulet; il ne s'écoule par conséquent que 10 à 12 heures entre le moment de l'innervation purement pneumogastrique et celui d'un système nerveux mixte (vague et sympathique).

La question la plus compliquée consiste à définir la nature des premières fibres cardiaques du vague. S'agirait-il ici, ainsi que l'un de nous (Szepsenwol et Baumann, 1935) l'a constaté chez la Truite, de fibres sensitives? Celles-ci pénétreraient tout d'abord dans l'organe pulsatile et ne seraient rejointes que plus tardivement par les fibres motrices ? Il est difficile, chez le Canard et le Poulet, de se prononcer catégoriquement; mais, en suivant pas à pas la formation de la dixième paire, on en a bien l'impression. Chez un embryon de Canard de 65 à 66 heures, alors que le ganglion commun du glosso-pharyngien-vague est déjà en pleine différenciation et aborde par sa racine centrale la vésicule cérébrale postérieure, la racine motrice n'existe pas encore; celle-ci n'apparaît qu'à 70 heures, sous forme de fibres radiculaires antérieures et postérieures (ces dernières sont considérées par BEccari comme constituant le nerf spinal). En se basant sur ce premier fait, il ne serait guẹre possible de trancher la question de la nature des premières fibres vagales du cœur, car le retard d'apparition de la racine motrice sur la sensitive est bien minime ( 2 heures) et, une fois les deux branches réunies, il est impossible de les différencier.

Un phénomène plus démonstratif se produit: au moment où, chez un embryon de Canard de 84 heures, la racine centrale mixte du vague (formée des fibres sensitives issues du ganglion jugulaire et des fibres motrices) aborde, par quelques filaments, le ganglion plexiforme thoracique, celui-ci, déjà en pleine différenciation, envoie par son extrémité périphérique un faisceau très fourni dans la direction du bulbe aortique; quelques fibrilles atteignent déjà 
les vaisseaux céphaliques (jugulaire et carotide); il est aisé d'en reconnaittre l'origine: ils proviennent manifestement du ganglion plexiforme. D'autre part, le fait que ces fibres se terminent dans la paroi vasculaire directement au contact de l'endothélium parle aussi en faveur de leur nature sensitive.

En somme, il semble bien que l'innervation primitive du cœur chez les Oiseaux (Canard et Poulet) est, tout au début, de nature sensitive. Quelle signification tirer de ce fait? Les fibres sensitives inciteraient-elles la croissance des éléments moteurs en transmettant aux centres supérieurs une excitation périphérique produite par les contractions du cœur? Dans ce cas, il y aurait une analogie avec les constatations que l'un de nous a faites sur la marche de la différenciation de l'are réflexe chez l'Axolotl (Szepsenwol, 1934); chez les Amphibiens, ce sont les éléments des ganglions craniens qui se différencient tout d'abord; c'est seulement lorsque leurs prolongements centraux pénètrent dans le rhombencéphale que débute la fibrillation des neurones moteurs.

En ce qui concerne l'origine des cellules intracardiaques, il semble bien qu'elle se trouve dans la chaîne du sympathique. De très bonne heure, soit déjà chez un embryon de Canard de quatre jours, on voit glisser le long de l'extrémité antérieure de l'aorte, à partir des ganglions rétro-aortiques, des cellules ayant déjà acquis des caractères nerveux distinctifs, qui se propagent dans la direction des vaisseaux branchiaux et de la paroi postérieure du pharynx. Des vaisseaux du cou, au pourtour desquels elles forment, avec les branches périphériques du vague, un plexus, on les voit se diriger vers le bulbe aortique: c'est là l'origine des éléments nerveux de la portion artérielle du cœur.

Pour le cœur veineux, les faits sont beaucoup moins précis. Nous avons bien constaté que c'est à partir du plexus intestinal qu'émigrent les éléments nerveux qu'on trouve dans la portion auriculaire du cœur entre 118 et 132 heures chez le Canard; mais cela ne nous renseigne pas sur leur nature, car la question de l'origine du plexus myentérique n'est pas encore élucidée; celui-ci apparaît d'emblée, chez un embryon de 86 à 88 heures, tout le long de l'extrémité supérieure du tractus intestinal; il n'est pas possible de trouver à des stades plus jeunes les signes de son origine et de sa différenciation; c'est pour cela que Tello a été amené à envisager la possibilité d'une différenciation sur place de cellules mésenchymateuses ou de 
cellules médullaires émigrées très précocement avant toute manifestation neurofibrillaire. C'est à cette deuxième hypothèse que Van Campenhout s'est rangé, en se basant sur des expériences de greffes chorio-allantoïdiennes que nous avons citées plus haut (seuls des fragments de tube digestif d'embryons de Poulet de plus de 56 heures donnent naissance à des plexus nerveux intraviscéraux).

Il nous semble aussi que les éléments nerveux myentériques ne se différencient pas à partir du mésenchyme; bien que nous n'ayons pas pu observer leur migration dans le tube digestif, nous sommes enclins à penser que celle-ci se produit tout de même, puisqu'au niveau du pharynx nous l'avons constatée. D'autre part, l'hypothèse que des cellules nerveuses émigreraient le long du nerf vague (Abel, Muller) nous semble très improbable; nulle part nous n'avons pu trouver, le long du pneumogastrique, une seule cellule de nature nerveuse.

En somme, d'après les faits que nous avons constaté, ce seraient les fibres vagales (sensitives) qui abordent en premier le système vasculo-cardiaque chez les Oiseaux (Canard de 84 heures et Poulet de 68 heures). Les cellules nerveuses, d'origine sympathique, pénétreraient plus tardivement (Canard de 104 heures et Poulet de 84 heures); elles proviendraient directement du ganglion cervical supérieur pour la portion ventriculaire et par l'intermédiaire du plexus intestinal pour la portion auriculaire.

\section{BIBLIOGRAPHIE}

1912. AвеL, W. Further observations on the development of the sympathetic nervous system in the chick. Journ. Anat. and Physiol., 47, p. 35-72.

1934. BAUMANn, A. Observations sur les premiers stades de l'innervation du cour chez un Batracien anoure (Bombinator pachypus Bonap.). Revue suisse de Zoologie, 41, No 12. p. 235-261.

1931. Campenhout, E. Van. Le développement du système nerveux sympathique chez le Poulet. Arch. de Biol., 42, p. 479-507.

1892. Couvreur, E. Le pneumogastrique des Oiseaux. Annales de l'Univ. de Lyon, 2, Fasc. 3, p. 1-104. 
1907. Froriep, A. Die Entwickelung und Bau des autonomen Nervensystems. Medizinisch-naturwiss. Arch., I, p. 301-321.

1908. Gerini, C. Quelques recherches sur les premières phases du développement des neurofibrilles primitives chez l'embryon de Poulet. Anat. Anz., 33, p. 178.

1890-92. His, W., jr. Ueber die Entwickelung des Sympathicus bei Wirbeltieren mit besonderer Berücksichtigung der Herzganglien. Verhandl. der anatomischen Gesellschaft, Wien, p. 29.

1907. Kонм, A. Ueber die Entwickelung des Sympathischen Nervensystems der Säugetiere. Arch. f. Mikr. Anat., 70, p. 266-317.

1929. Kuntz, A. The autonomic nervous system. Lea and Febiger, Philadelphia.

1920. Müller, E. Beiträge zur Kenntnis des autonomen Nervensystems. I. Ueber die Entwickelung des Sympathicus und des Vagus bei den Selachiern. Arch. f. mikr. Anat., 94, p. 208-247.

1924. Perman, E. Anatomische Untersuchungen über die Herznerven bei den höheren Säugetieren und beim Menschen. Zeitschr. f. Anat. und Entwicklungsgeschichte, 71, p. 382-457.

1929. Szantroch, Z. L'histogenèse des ganglions nerveux du cœur. Bull. intern. Ac. Polon., Cracovie, 2, No 8-10, p. 417-431.

1934. Szepsenwol, J. La causalité de la différenciation neuronale chez les Batraciens. C.R. de la Soc. de Biol., CXVII, p. 305-306.

1935. _ Le développement primitif de la glande carotidienne chez les embryons de Canard. C.R. de la Soc. de Biol., t. 119, p. 13-15.

1935. — Sur l'origine du glomus caroticum chez les embryons d'Oișeaux. C.R. du Congrès de l'Assoc. des Anatomistes, Montpellier, 1935.

1935. — et Baumann, A. Sur la nature des premières fibres nerveuses qui pénètrent dans le cœur chez un poisson Téléostéen (Trutta fario). Revue suisse de Zoologie, 42, No 3, p. 39-49.

1921. Tello, J. F. Les différenciations neuronales dans l'embryon de Poulet pendant les premiers jours d'incubation. Trav. du Lab. de Rech. Biol., Madrid, 21.

1924. — La précocité embryonnaire du plexus d'Auerbach et ses différences dans les intestins antérieur et postérieur. Trav. du Lab. de Rech. Biol., Madrid, 22.

1924. — Développement et terminaison du nerf dépresseur. Trav. du Lab. de Rech. Biol., Madrid, 22, p. 295-308.

1927. Uchid a, S. Ueber die Entwickelung des sympathischen Nervensystems. Acta Schol. Med. Univ. Imp., Kioto, 10, p. 63-136. 

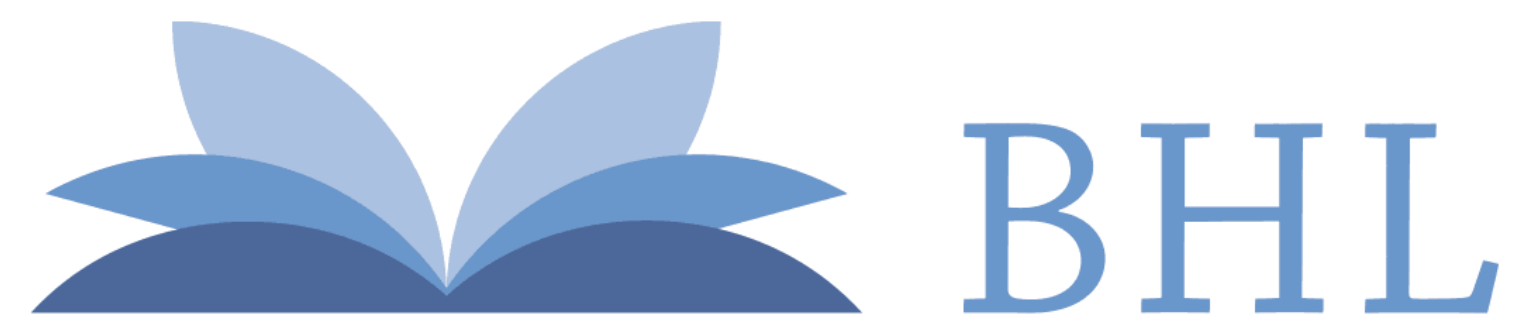

\section{Biodiversity Heritage Library}

Szepsenwol, J and Guinand-Bron, Alix. 1936. "L'origine et la nature de I'innervation primitive du coeur chez les embryons d'Oiseaux (Canard et Poulet)." Revue suisse de zoologie 43, 1-23.

https://doi.org/10.5962/bhl.part.117679.

View This Item Online: https://www.biodiversitylibrary.org/item/148563

DOI: https://doi.org/10.5962/bhl.part.117679

Permalink: https://www.biodiversitylibrary.org/partpdf/117679

\section{Holding Institution}

American Museum of Natural History Library

\section{Sponsored by}

BHL-SIL-FEDLINK

\section{Copyright \& Reuse}

Copyright Status: In copyright. Digitized with the permission of the rights holder.

Rights Holder: Muséum d'histoire naturelle - Ville de Genève

This document was created from content at the Biodiversity Heritage Library, the world's largest open access digital library for biodiversity literature and archives. Visit BHL at https://www.biodiversitylibrary.org. 